\title{
A NOTE ON THE LOWER SEMI-CONTINUITY OF DOUBLE INTEGRALS IN THE PARAMETRIC FORM
}

\author{
WILLIAM SCOTT
}

Consider a double integral problem of the calculus of variations in parametric form, that is, a problem involving an integral of the form

$$
\iint_{B} f(x, X) d u
$$

where $x$ stands for the three coordinate functions, $x^{1}\left(u^{1}, u^{2}\right), x^{2}\left(u^{1}, u^{2}\right)$, $x^{3}\left(u^{1}, u^{2}\right)$, and $X$ stands for their three Jacobians.

Various theorems of the following general type have been proved by McShane, ${ }^{1}$ Caccioppoli, ${ }^{2}$ Cimmino, ${ }^{3}$ and Radó ${ }^{4}$

If (i) the triples of functions $x_{n}^{i}\left(u^{1}, u^{2}\right), i=1,2 ; n=0,1,2, \cdots$, satisfy certain conditions; (ii) the surfaces defined by $x^{i}=x_{n}^{i}\left(u^{1}, u^{2}\right)$, $i=1,2 ; n=1,2, \cdots$, converge in some prescribed sense to the surface defined by $x_{0}^{i}=x_{0}^{i}\left(u^{1}, u^{2}\right), i=1,2$; (iii) $f(x, X)$ satisfies certain conditions; then

$$
\liminf _{n} \iint_{B_{n}} f\left(x_{n}(u), X_{n}(u)\right) d u \geqq \iint_{B_{0}} f\left(x_{0}(u), X_{0}(u)\right) d u .
$$

The conditions on $f(x, X)$ usually contain a condition on its Weierstrass $E$-function

$$
E(x, X, \bar{X})=f(x, \bar{X})-\bar{X}^{\alpha} f_{\alpha}(x, X),
$$

the summation convention being used for convenience in writing.

McShane $^{1}$ requires either $f>0, E \geqq 0$; or $f \geqq 0, E>0$. Caccioppoli ${ }^{2}$ makes the same requirements. Cimmino ${ }^{3}$ requires only $f \geqq 0, E \geqq 0$, but works with smooth surfaces.

Presented to the Society, April 11, 1941 under the title A lemma on the Weierstrass E-function; received by the editors January 17, 1942.

1 McShane, On the semi-continuity of double integrals in the calculus of variations, Annals of Mathematics, (2), vol. 33 (1932), pp. 460-484.

${ }^{2}$ Caccioppoli, Gli integrali doppi di forma parametrica nel calcolo delle variazioni, Arri del Reale Istituto Veneto di Scienze, Lettere ed Arti, Anno Accademico, 1933 1934, vol. 93, Part 2.

${ }^{3}$ Cimmino, Sulle condizione necessarie e sufficienti per la semi-continuita degle integrali doppi di forma parametrica, Annali di Mathematica Pura ed Applicata, vol. 15 (1936), pp. 159-173. The author wishes to thank Professor McShane for calling his attention to this paper.

${ }^{4}$ Rad6, On the semi-continuity of double integrals in the parametric form, Transactions of this Society, vol. 51 (1942), pp. 336-361. The author wishes to thank Professor Rado for access to the manuscript of this paper before it was published. 
Radó $\delta^{4}$ proves the semi-continuity theorems under the same restrictions on $f(x, X)$ as McShane and Caccioppoli, but for a more general class of surfaces; namely, the class of surfaces that admit of a parametric representation for which the Lebesgue area is given by the usual integral formula.

The question arises as to whether we cannot prove the semi-continuity theorem for the class of surfaces which Radó considers with the requirements that $f \geqq 0, E \geqq 0$. We shall first prove a lemma on the Weierstrass $E$-function, then prove a stronger form of Lemma 1.5 in Radó. ${ }^{4}$ The remainder of the proof as given by Radó will then prove the semi-continuity theorem with $f \geqq 0, E \geqq 0$ if the revised form of Lemma 1.5 is used.

We shall use the notations and definitions in $\operatorname{Rado}^{4}$ throughout the remainder of the paper.

Lemma 1. If $f$ is admissible and $x_{0}, X_{0}$ are such that

(1) $f\left(x_{0}, X_{0}\right)>0$,

(2) $f\left(x_{0}, X\right) \geqq 0$,

(3) $E\left(x_{0}, X_{0}, X\right) \geqq 0$,

for every $X$, then there exists a $\delta>0$ such that if $\bar{X}^{\alpha} f_{\alpha}\left(x_{0}, X_{0}\right)>0$ then $f\left(x_{0}, \bar{X}\right)>\delta\|\bar{X}\|$.

Proof. Since

$$
X_{0}^{\alpha} f_{\alpha}\left(x_{0}, X_{0}\right)=f\left(x_{0}, X_{0}\right)>0
$$

at least one of $f_{1}\left(x_{0}, X_{0}\right), f_{2}\left(x_{0}, X_{0}\right), f_{3}\left(x_{0}, X_{0}\right)$ is different from 0 . Hence the set of points $\bar{X}$ satisfying $\bar{X}^{\alpha} f_{\alpha}\left(x_{0}, X_{0}\right)>0,\|\bar{X}\|=1$, is an open hemisphere $H^{0}$. Since $E\left(x_{0}, X_{0}, \bar{X}\right) \geqq 0$ we have $f\left(x_{0}, \bar{X}\right)>0$ for every $\bar{X} \in H^{0}$. If we deny the lemma, then there exists a sequence $\left\{\bar{X}_{n}\right\}$, $\bar{X}_{n} \in H^{0}$ such that $f\left(x_{0}, \bar{X}_{n}\right) \rightarrow 0$. Hence there exists a point $\bar{X}_{0} \in H-H^{0}$ such that $f\left(x_{0}, \bar{X}_{0}\right)=0$.

Let $H-H^{0}=C$, and let the plane containing $C$ be $P$. Let $d(\bar{X}, P)$ denote the distance of $\bar{X}$ from $P$. Then if $\bar{X} \in H$ we have

$$
\bar{X}^{\alpha} f_{\alpha}\left(x_{0}, X_{0}\right)=d(\bar{X}, P)\left(\sum_{i=1}^{3} f_{i}^{2}\left(x_{0}, X_{0}\right)\right)^{1 / 2} .
$$

Let $C^{\prime}$ be the great circle with center at $X=0$, unit radius, passing through $\bar{X}_{0}$, and perpendicular to $C$. Let the direction cosines of the tangent to $C^{\prime}$ at $\bar{X}_{0}$ toward $H^{0}$ be $\cos \phi_{1}, \cos \phi_{2}, \cos \phi_{3}$.

Let $\partial f\left(x_{0}, \bar{X}_{0}\right) / \partial \nu$ be the directional derivative of $f$ along this tangent. Then we have

$$
\frac{\partial f\left(x_{0}, \bar{X}_{0}\right)}{\partial \nu}=f_{\alpha}\left(x_{0}, \bar{X}_{0}\right) \cos \phi_{\alpha} .
$$


On the one hand, we have from (*)

$$
\frac{\partial f\left(x_{0}, \bar{X}_{0}\right)}{\partial \nu} \geqq\left(\sum_{i=1}^{3} f_{i}^{2}\left(x_{0}, X_{0}\right)\right)^{1 / 2}>0,
$$

and on the other hand, since $f\left(x_{0}, \bar{X}_{0}\right)=0, f\left(x_{0}, X\right) \geqq 0$ we have

$$
\frac{\partial f\left(x_{0}, \bar{X}_{0}\right)}{\partial \nu}=0
$$

This is a contradiction. Hence the lemma is true.

LEMMA 2. Let there be given an admissible $f$ and a set of six constants $\left(x_{0}^{1}, x_{0}^{2}, x_{0}^{3}, X_{0}^{1}, X_{0}^{2}, X_{0}^{3}\right)=\left(x_{0}, X_{0}\right)$ such that

(a) $f\left(x_{0}, X_{0}\right)>0$,

(b) $f\left(x_{0}, X\right) \geqq 0$,

(c) $E\left(x_{0}, X_{0}, X\right) \geqq 0$,

for all $X$. Then there exist two positive constants $\delta_{1}, \delta_{2}$ such that the following holds. If $\eta, \bar{x}^{1}, \bar{x}^{2}, \bar{x}^{3}, \bar{X}^{1}, \bar{X}^{2}, \bar{X}^{3}$ satisfy the conditions

( $\alpha) 0<\eta<\delta_{1}$,

(及) $\bar{X}^{\alpha} f_{\alpha}\left(x_{0}, X_{0}\right)>0$,

$(\gamma)\left|f(\bar{x}, \bar{X})-f\left(x_{0}, \bar{X}\right)\right| \leqq \eta\|\bar{X}\|$,

then

$$
f(\bar{x}, \bar{X})-f\left(x_{0}, \bar{X}\right)+E\left(x_{0}, X_{0}, \bar{X}\right) \geqq-\eta \delta_{2} \bar{X}^{\alpha} f_{\alpha}\left(x_{0}, X_{0}\right) .
$$

Proof. The conditions of Lemma 1 are satisfied. Hence there exists a positive constant $\delta$ as described there. Let $\delta_{1}=\delta / 2, \delta_{2}=2 / \delta$.

Case 1. $\bar{X}^{\alpha} f_{\alpha}\left(x_{0}, X_{0}\right) \geqq(\delta / 2)\|\bar{X}\|$. Then

$$
\begin{aligned}
f(\bar{x}, \bar{X})-f\left(x_{0}, \bar{X}\right)+E\left(x_{0}, X_{0}, \bar{X}\right) & \geqq-\eta\|\bar{X}\| \\
& \geqq-\eta \delta_{2} \bar{X}^{\alpha} f_{c:}\left(x_{0}, X_{0}\right) .
\end{aligned}
$$

Case 2. $\bar{X}^{\alpha} f_{\alpha}\left(x_{0}, X_{0}\right)<(\delta / 2)\|\bar{X}\|$. Then

$$
\begin{aligned}
& f(\bar{x}, \bar{X})-f\left(x_{0}, \bar{X}\right)+E\left(x_{0}, X_{0}, \bar{X}\right) \\
& \\
&=f(\bar{x}, \bar{X})-\bar{X}^{\alpha} f_{\alpha}\left(x_{0}, X_{0}\right) \\
& \geqq f\left(x_{0}, \bar{X}\right)-\eta\|\bar{X}\|-\bar{X}^{\alpha} f_{\alpha}\left(x_{0}, X_{0}\right) \\
& \geqq 0 \\
& \geqq-\eta \delta_{2} \bar{X}^{\alpha} f_{a}\left(x_{0}, X_{0}\right) .
\end{aligned}
$$

Therefore the lemma is true.

Let $K_{2}$ be the class of oriented surfaces whose Lebesgue area is given by the usual integral formula. Let ${ }_{o} S_{0},{ }_{o} S_{n}$ be oriented surfaces in the class $K_{2}$ such that: 
(I) ${ }_{0} S_{n} \rightarrow{ }_{0} S_{0}$.

(II) If $T_{0}: x_{0}^{i}=x_{0}^{i}\left(u^{1}, u^{2}\right),\left(u^{1}, u^{2}\right) \in B_{0}, i=1,2,3$ is a representation of ${ }_{o} S_{0}$ then there exists, in $x$-space, a closed bounded set $A$ such that $\sum\left(T_{0}, B_{0}\right) \subset A^{0}$, and $f(x, X) \geqq 0$ for $x \in A$ and for every vector $X$.

(III) For a. e. point $u \in B_{0}^{0}$ such that $X_{0}(u)$ exists and is different from 0 we have $E\left(x_{0}(u), X_{0}(u), \bar{X}\right) \geqq 0$ for every vector $\bar{X} \neq 0$.

As we mentioned above the proof given by Radó will now lead to the following result.

THEOREM. If $f$ is admissible and if conditions (I), (II), and (III) are satisfied then

$$
\underset{n}{\lim \inf } \iint_{B_{n}} f\left(x_{n}(u), X_{n}(u)\right) d u \geqq \iint_{B_{0}} f\left(x_{0}(u), X_{0}(u)\right) d u .
$$

This theorem is final in a definite sense. We cannot enlarge the class of surfaces if we use ordinary Jacobians. Counterexamples have been given which show the necessity of the conditions $E \geqq 0, f \geqq 0$. Moreover if $f$ is only assumed to be continuous, convex, and nonnegative, then we do not necessarily have lower semi-continuity. For, let

$$
f(x, X)=\left|X^{3}+g\left(x^{1}, x^{3}\right) X^{1}\right|
$$

where

$$
\begin{aligned}
g\left(x^{1}, x^{3}\right)= & \frac{\cos 16 x^{1}}{2} \\
g\left(x^{1}, x^{3}\right)= & \frac{\cos n^{4} x^{1}}{n} \quad \text { if } 1 / n-1 / n^{3} \leqq x^{3} \leqq 1 / n+1 / n^{3}, n \geqq 2, \\
g\left(x^{1}, x^{3}\right)= & \frac{\cos (n+1)^{4} x^{1}}{n+1} \\
& +\frac{x^{3}-\left(1 /(n+1)+1 /(n+1)^{3}\right)}{\left(1 / n-1 / n^{3}\right)-\left(1 /(n+1)+1 /(n+1)^{3}\right)} \\
& \cdot\left[\frac{\cos n^{4} x^{1}}{n}-\frac{\cos (n+1)^{4} x^{1}}{n+1}\right] \\
g\left(x^{1}, x^{3}\right)= & 0 \quad \text { if } 1 /(n+1)+1 /(n+1)^{3}<x^{3}<1 / n-1 / n^{3},
\end{aligned}
$$

Then $f$ satisfies all the conditions stated. Further let

$$
x_{0}^{1}=u^{1}, \quad x_{n}^{1}=u^{1}, \quad 0 \leqq u^{1} \leqq \pi,
$$




$$
\begin{aligned}
& x_{0}^{2}=u^{2}, \quad x_{n}^{2}=u^{2}, \\
& 0 \leqq u^{2} \leqq 1 \\
& x_{0}^{3}=0, \quad x_{n}^{3}=\frac{1}{n}+\frac{\sin n^{4} u^{1}}{n^{3}} \text {. }
\end{aligned}
$$

Then we have

$$
\begin{aligned}
\liminf _{n} \iint_{B_{n}} f\left(x_{n}, X_{n}\right) d u & =\liminf \int_{n}^{\pi} \int_{0}^{1}\left|1-\cos ^{2} n^{4} u^{1}\right| d u^{2} d u^{1} \\
& =\frac{\pi}{2}<\pi=\int_{0}^{\pi} \int_{0}^{1} d u^{2} d u^{1} \\
& =\iint_{B_{0}} f\left(x_{0}, X_{0}\right) d u .
\end{aligned}
$$

The Ohio State University

\section{A NON-INVOLUTORIAL SPACE TRANSFORMATION ASSOCIATED WITH A $Q_{1, n}$ CONGRUENCE}

\section{L. VEST}

1. Introduction. The involutorial transformation associated with the congruence of lines meeting a curve of order $m$ and an $(m-1)$ fold secant has been studied by DePaolis, ${ }^{1}$ and $\mathrm{Vogt}^{2}$ has studied the non-involutorial transformations for a linear congruence and bundle of lines. Cunningham ${ }^{3}$ has recently studied some non-involutorial transformations associated with a $Q_{1,2}$ congruence. In the present paper a non-involutorial transformation associated with the congruence of lines on a plane curve of order $n$ having an $(n-1)$-point and a secant through that point is considered. The bundle of lines through the multiple point is not considered as belonging to the congruence. The tangents to the curve at the point are considered to be distinct.

Received by the editors November 26, 1941.

${ }^{1}$ DePaolis, Alcuni particolari transformazioni involutori dello spazio, Rendiconti dell'Academia dei Lincei, Rome, (4), vol. 1 (1885), pp. 735-742, 745-758.

${ }^{2}$ Vogt, Zentrale und windschiefe Raum-Verwandtschaften, Jahresbericht der Schlesischen Gesellschaft für Vaterlandische Kultur, class 84, 1906, pp. 8-16.

${ }^{3}$ Cunningham, Non-involutorial space transformations associated with a $Q_{1,2}$ congruence, this Bulletin, vol. 47 (1941), pp. 309-312. 